\title{
Effect of Water Absorption on Interaction between Matrix Cracking and Fiber Bridging for Stress Corrosion Cracking of PMC*
}

\author{
Akira KOBIKI** and Hiroyuki KAWADA***
}

\begin{abstract}
The effect of water on fiber bridging of polymer matrix composites (PMCs) has been studied for stress-corrosion cracking (SCC). The fiber bridging during crack propagation in the PMC was observed directly using a transparent polymer matrix. Specimens were immersed and weighed just before conducting the crack propagation test. The contribution of mechanical degradation caused by the water to the bridging was measured as a function of weight gain due to water absorption. The critical energy release rate of the matrix decreased with increase of the water absorption. The energy release rate of a bridged crack varied with the water absorption. To investigate the variation in the bridging mechanism under the water environment, we calculated the bridging stress the $\sigma_{\mathrm{t}}$ and the bridging contribution to crack propagation resistance $\Delta G$ based on a single-fiber bridging mechanism with the interfacial debonding length and the interfacial debonding energy. The debonding length was measured directly, and the debonding energy was obtained in a fragmentation test. The calculation showed that the increase in the interfacial debonding length influenced the fiber bridging mechanism.
\end{abstract}

Key Words: GFRP, Fiber Bridging, Water Absorption, Interfacial Debonding

\section{Introduction}

PMCs have been used in some hostile environmental applications due to their good environmental resistance and their high specific strength and stiffness. Generally, PMCs used in corrosive environments are reinforced by glass fibers $^{(1)-(5)}$. However, fibers in the matrix are corroded in such environments. Studies on SCC of PMCs in our laboratory have revealed that the SCC of the PMCs was also promoted under the water environment, although the fibers were not obviously corroded by water ${ }^{(5)}$.

According to fracture mechanics, fiber bridging enhances the toughness of a composite. The fiber bridging is associated with interfacial debonding and fiber pull out. Therefore, interfacial strength is an essential factor in the fiber bridging in the composite. Previously, we investigated an interfacial degradation caused by water ab-

* Received 27th May, 2005 (No. 05-4117)

** Graduate School of Waseda University, 3-4-1 Okubo, Shinjuku, Tokyo 169-8555, Japan. E-mail: kobiki@fuji.waseda.jp

*** Department of Mechanical Engineering, Waseda University, 3-4-1 Okubo, Shinjuku, Tokyo 169-8555, Japan sorption in a single-fiber composite ${ }^{(6)}$. The result showed that the water absorption obviously affected the interfacial degradation. Moreover, interfacial degradation was described as a function of moisture concentration at the fiber/matrix interface.

Fuller et al. measured the enhanced toughness obtained by a single fiber bridging by the DCDC test ${ }^{(7),(8)}$, which is useful to evaluate this kind of bridging. Sun et al. measured bridging-fiber stress by direct measurements of interfacial debonding lengths ${ }^{(9)}$. Xu et al. estimated the fiber-bridging mechanism on a critical energy release rate theoretically ${ }^{(10)}$. The experimental technique developed by Sun et al. is very useful to determine the relationship between fiber bridging and interfacial debonding in the composite.

In this study, we investigated the variation in the enhanced toughness obtained by fiber bridging under the water environment using the DCDC test ${ }^{(11)-(13)}$. Additionally, we conducted direct measurements of interfacial debonding to estimate fiber stress using the interfacial debonding energy, which has been described as a function of the moisture concentration. The estimation based on the interfacial debonding allowed us to determine the rela- 
tionship between the enhanced toughness and the moisture concentration.

\section{Experiment}

\subsection{DCDC test}

Observation of the crack tip is required to determine enhanced toughness. In the composite it is difficult to observe the crack tip directly for measurements of interfacial debonding. Therefore, we employed an experimental technique using a transparent polymer matrix according to Sun's technique ${ }^{(9)}$. Furthermore, a single-fiber bundle was embedded in the polymer to observe the crack tip directly. However, the conventional testing method for measurement of fracture toughness was not suitable for a specimen with the single-fiber bundle, because the polymer was too brittle to obtain stable crack-propagation. The DCDC test is a method to evaluate fracture toughness of such brittle materials, and was considered by Janssen ${ }^{(7)}$ and many subsequent authors to measure toughness of brittle materials $^{(8),(11)-(13)}$. In particular, Fuller et al. investigated an interaction between a single fiber and crack-propagation in a CMC using the DCDC test. When the DCDC test specimen is loaded in compression, two cracks initiate from the center-drilled hole and propagate parallel to the applied stress along the specimens mid-plane. Therefore we embedded two fiber bundles in a DCDC specimen for observation of two-fiber bridging.

\subsection{Experiment procedure}

Figure 1 shows the geometry and dimensions of the DCDC test specimen in the present experiment. ECRglass fibers with silane-coupling agent are employed, because they have the same mechanical properties as the E-glass fibers and are almost equal to the C-glass fibers in acid resistance. We prepared two types of specimens. Vinyl ester and epoxy were used for the matrices. The number of ECR-glass fibers was about 300. Table 1 shows the mechanical properties of each constituent prepared for the specimen. The two fiber bundles located as shown in Fig. 2 were embedded so as to be perpendicular to the crack-propagation direction. As mentioned above, since

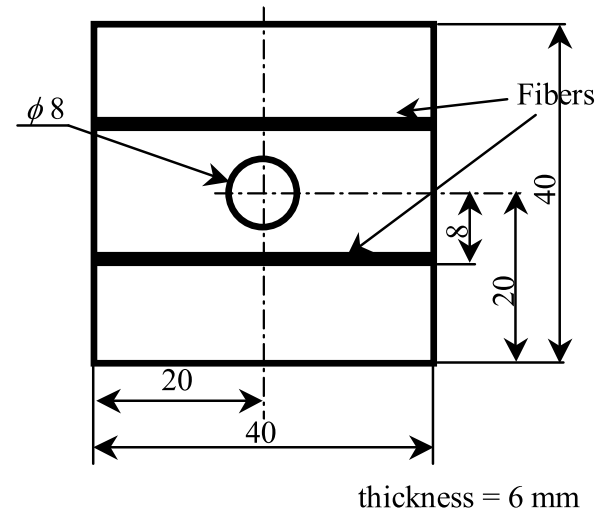

Fig. 1 Specimen geometry in DCDC test the two cracks can be observed in the DCDC test, the two fiber bundles are embedded. The specimens were immersed in purified water at $60^{\circ} \mathrm{C}$ from 0 to 672 hours just before conducting the DCDC test. During immersion, we periodically weighed the specimens and recorded the amount of water uptake as a function of time. All DCDC tests were performed under displacement control operated at a constant cross-head speed of $0.5 \mathrm{~mm} / \mathrm{min}$. The crack tip shape was observed by microscope to measure crack length. In the specimen with fiber bundles, the interfacial debonding length was measured by in-situ observation using photographing and video recording methods without unloading the specimen.

\section{Results and Discussion}

\subsection{Degradation of matrix toughness}

Figure 2 shows the amount of water uptake as a function of immersion time for both the vinyl ester and epoxy specimens. The percent weight gain was calculated as

$$
\frac{W_{\text {wet }}-W_{\text {dry }}}{W_{\text {dry }}} \times 100
$$

where $W_{\text {wet }}$ is the weight of the absorbed specimen and $W_{\text {dry }}$ is the weight of the dry specimen. The time to reach the saturation level was 20 hours $^{0.5}$ for the vinyl ester specimen. For the epoxy, the weight change did not reach the saturation level within the time range.

The crack propagated stably during the DCDC test. In other words, its crack-propagation velocity was controlled by the cross-head speed of the testing machine. To quantify crack-propagation behavior, an energy release rate, $G$, was calculated at the crack tip.

Table 1 Mechanical properties of materials

\begin{tabular}{c|c|c|c}
\hline Material & ECR-glass fiber & Vinyl ester & Epoxy \\
\hline Young's modulus & $78 \mathrm{GPa}$ & $3.6 \mathrm{GPa}$ & $4.0 \mathrm{GPa}$ \\
\hline Poisson's ratio & 0.15 & 0.30 & 0.30
\end{tabular}

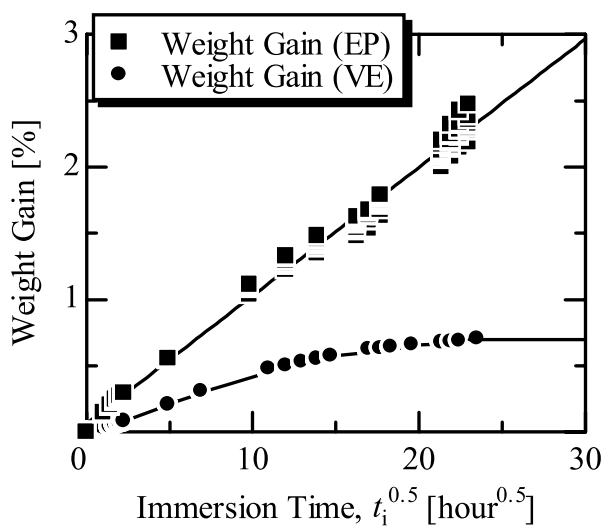

Fig. 2 Water absorption rate as a function of root of immersion time for vinyl ester and epoxy 


$$
G=\frac{\left(1-v^{2}\right)}{E}\left(K_{\mathrm{I}}^{2}+K_{\mathrm{II}}^{2}\right)
$$

We obtained the $G$ using Eq. (2) with the assumption that a crack propagated under only mode I. $K_{\mathrm{I}}$ is described as $K_{\mathrm{I}}=f(c / r) \sigma \sqrt{c}$, where $c$ is the crack length, $r$ is the radius of the drilled hole of the DCDC specimen and $f(c / r)$ is a geometry function of the specimen geometry. We defined the crack length at a mid-point of the crack surface through a given thickness. We can obtain the $f(c / r)$ by experimental calibration using a specimen of a known toughness. Therefore, we obtained the fracture toughness of the vinyl ester by Double Torsion (DT) test, the result of which showed the fracture toughness, $K_{\mathrm{IC}}=0.87 \mathrm{MPa} \sqrt{\mathrm{m}}$. Finally, on the basis of the relationship between $\sigma_{\text {app }}, c$ and $K_{\mathrm{IC}}=0.87 \mathrm{MPa} \sqrt{\mathrm{m}}$, the $f(c / r)$ is described as follows,

$$
\begin{gathered}
f(c / r)=-0.0580\left(\frac{c}{r}\right)^{3}+0.3129\left(\frac{c}{r}\right)^{2} \\
-0.5860\left(\frac{c}{r}\right)+0.4627 \\
(0.75 \leq c / r \leq 2)
\end{gathered}
$$

Using Eqs. (2) and (3), we obtained an energy release rate of a matrix, $G_{\text {mat }}$. Young's modulus of each matrix was assumed to be constant against the water uptake ${ }^{(6)}$. Figure 3 shows the relationship between the immersion time and the $G_{\text {mat }}$. The $G_{\text {mat }}$ of the epoxy specimen was higher than that of the vinyl ester at $t_{\mathrm{i}}=0$ hours ${ }^{0.5}$. The vinyl ester was saturated with the water at 21 hours ${ }^{0.5}$, and the $G_{\text {mat }}$ of the vinyl ester converged at $171 \mathrm{~J} / \mathrm{m}^{2}$ after 10 hours ${ }^{0.5}$. On the other hand, the $G_{\text {mat }}$ of the epoxy specimen converged at $153 \mathrm{~J} / \mathrm{m}^{2}$ after 15 hours $^{0.5}$. Therefore a crossing point of the $G_{\text {mat }}$ existed at 10 hours ${ }^{0.5}$ in this experiment.

Degradation of the $G_{\text {mat }}$ under the water environment converged earlier than the saturation of the water uptake. The degradation is considered to depend on the moisture concentration distribution for each matrix. In the DCDC test, the crack propagation initiated from the edge of the

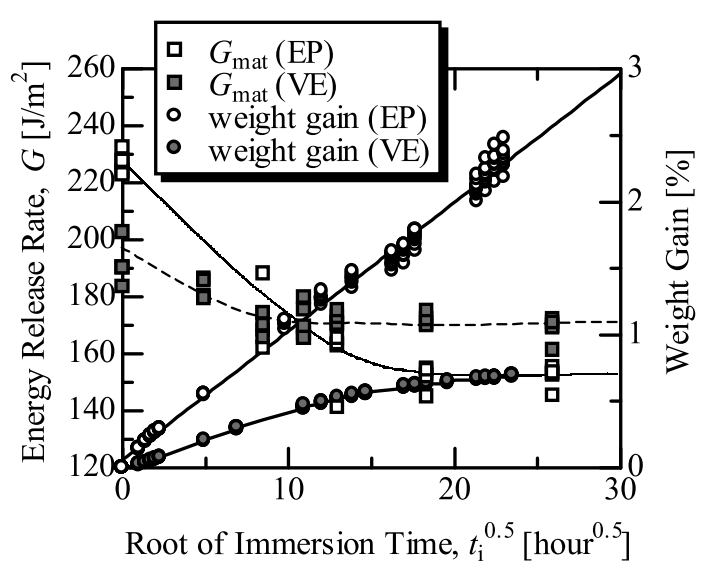

Fig. 3 Critical energy release rate of matrix and water absorption rate as a function of root of immersion time for vinyl ester and epoxy specimen drilled hole. The edge is exposed in the water, so its the moisture concentration is relatively large in the DCDC specimen, and the toughness at the crack tip is degraded relatively early.

\subsection{Fiber bridging}

Figure 4 (a) is a photograph of the crack tip when the crack passed the fiber bundle. The crack was bridged by the fiber bundle as shown in Fig. 4 (b) after crack bowing. When the fiber bridging was observed in the experiments, the crack-tip was defined similarly to the matrix-crack tip. During the bridging, the applied energy release rate $G_{\text {bri }}$ was calculated using the same equations with the $G_{\text {mat }}$. Figure 5 shows the $G_{\text {mat }}$ and the $G_{\text {bri }}$ as a function of the immersion time. The $G_{\text {bri }}$ is divided into the $G_{\text {mat }}$ and a term obtained by fibers, $\Delta G$. Figure 6 shows the relationship between the moisture-concentration distribution at the fiber position in the specimen and the immersion time in the ECR/vinyl ester specimen obtained from an FEM analysis based on Fickian diffusion. The diffusion constant was determined as $6.0 \times 10^{-6} \mathrm{~mm}^{2} / \mathrm{sec}$ from the relationship between the water uptake and the immersion time. The $\Delta G$ reaches its peak at 6 hours $^{0.5}$ in Fig. 6 . The FEM-analysis result shows that the moisture concentration at the fiber increased after 6 hours ${ }^{0.5}$, and this moisture enhanced the $\Delta G$ at 6 hours $^{0.5}$, although the $\Delta G$ decreased

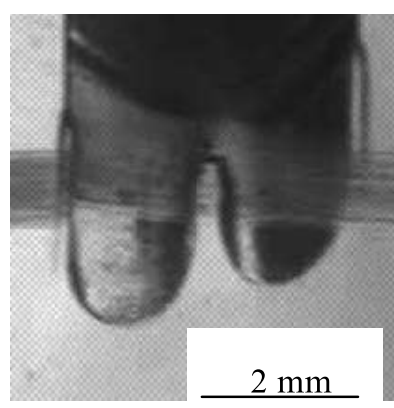

(a) Bowing

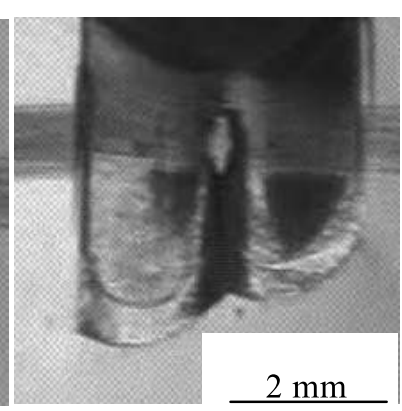

(b) Bridging
Fig. 4 Photograph of crack tip shape obtained by in-situ measurements

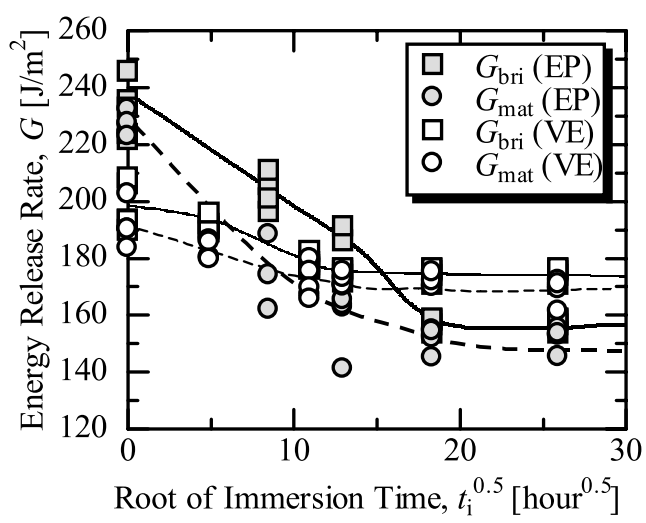

Fig. 5 Critical energy release rate of matrix and bridged specimen as a function of root of immersion time 


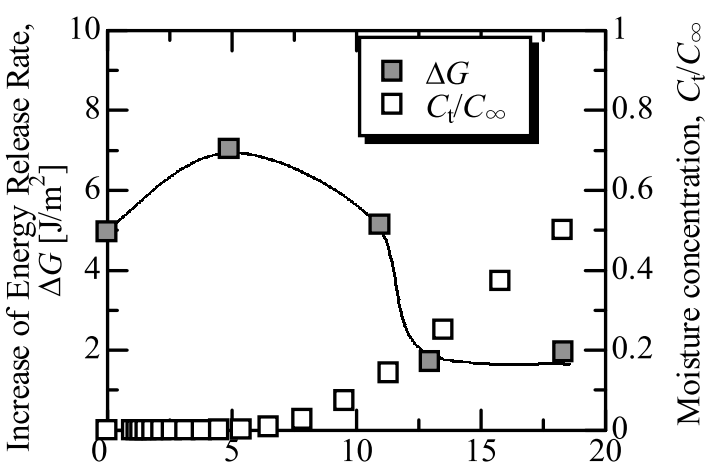

Root of Immersion Time, $t_{\mathrm{i}}^{0.5}$ [hour ${ }^{0.5}$ ]

Fig. 6 Increase of energy release rate $\Delta G$ and moisture concentration of fiber as a function of immersion time

after 6 hours ${ }^{0.5}$. The $\Delta G$ converged on $136 \mathrm{~J} / \mathrm{m}^{2}$ after 12 hours ${ }^{0.5}$. This convergence is considered to be an essential factor affecting the threshold properties in the SCC.

The $\Delta G$ variation under water environment is determined by the bridging mechanism associated with the interfacial debonding. The previous study showed that interfacial degradation depended on the moistureconcentration $^{(6)}$. Therefore, the interfacial degradation due to the increase in moisture concentration was predicted to influence the toughness obtained by the fiber bridging as shown in Fig. 6.

\section{Toughness Enhancement by Fiber Bridging}

\section{1 Determination of $\Delta G$}

For discussing the cause of the $\Delta G$ variation, the toughening mechanism is considered on the basis of microscopic fracture mechanics. The fiber stress distribution is required for determination of the $\Delta G$. The stress distribution was obtained under the assumption that the stress caused the interfacial debonding observed by direct measurements. We determined the $\Delta G$ as follows ${ }^{(10)}$,

$$
\Delta G=2 V_{\mathrm{f}} \int(1-q) \sigma_{\mathrm{t}}(u) d u
$$

where $\sigma_{\mathrm{t}}$ is the fiber-stress on the crack surface, $q$ is the failure probability function, and $u$ is half of the crack opening displacement. It was considered that some of the fibers would fail with increase of the stress applied to the fiber. Therefore the fiber failure is described by a failure probability function ${ }^{(14)}$. The $\sigma_{\mathrm{t}}$ is considered with microscopic mechanics. The fiber/matrix interfacial debonding/sliding has been studied theoretically, while Sun and Singh also examined it experimentally ${ }^{(9)}$. In the model, the $\sigma_{\mathrm{t}}$ is described as follows,

$$
\sigma_{\mathrm{t}}=\frac{2 E_{\mathrm{c}} \tau_{\mathrm{f}} L_{\mathrm{d}}}{r_{\mathrm{f}} V_{\mathrm{m}} E_{\mathrm{m}}}+\frac{2 E_{\mathrm{c}} \tau_{\mathrm{f}}}{E_{\mathrm{m}} V_{\mathrm{m}} \rho}+\sqrt{\frac{4 E_{\mathrm{f}} E_{\mathrm{c}} \Gamma_{\mathrm{d}}}{r_{\mathrm{f}} E_{\mathrm{m}} V_{\mathrm{m}}}}
$$

where $L_{\mathrm{d}}$ is the interfacial debonding length, $r_{\mathrm{f}}$ is the radius of a fiber, $\tau_{\mathrm{f}}$ is the interfacial frictional stress, $E_{\mathrm{f}}, E_{\mathrm{m}}$ is the Young's modulus of a fiber and one of a matrix respectively, $V_{\mathrm{f}}, V_{\mathrm{m}}$ is the volume fraction of the fiber and

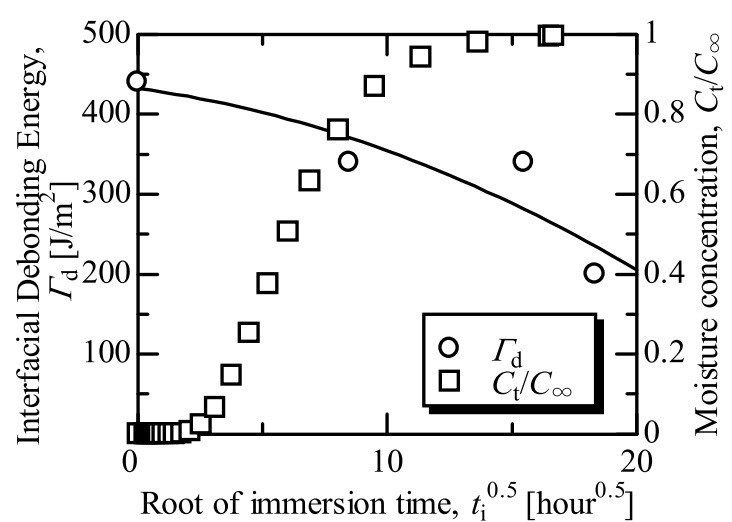

Fig. 7 Interfacial debonding energy obtained by fragmentation test as a function of root of immersion time and corresponding moisture concentration

matrix, respectively, $E_{\mathrm{c}}$ is described as $E_{\mathrm{c}}=E_{\mathrm{f}} V_{\mathrm{f}}+E_{\mathrm{m}} V_{\mathrm{m}}$, while

$$
\rho^{2}=\frac{4 E_{\mathrm{c}} G_{\mathrm{m}}}{V_{\mathrm{m}} E_{\mathrm{m}} E_{\mathrm{f}} \phi},
$$

and

$$
\phi=-\frac{2 \ln V_{\mathrm{f}}+V_{\mathrm{m}}\left(3-V_{\mathrm{f}}\right)}{2 V_{\mathrm{m}}^{2}} .
$$

Here $V_{\mathrm{f}}$ is determined to be $2 \%$ from an observation of the crack surface. $\tau_{\mathrm{f}}$ was assumed as $5 \mathrm{MPa}$, and $r_{\mathrm{f}}$ of the ECR-glass fiber is $12.25 \mu \mathrm{m}$. Subsequently, the failure probability function is described as,

$$
q=1-\exp \left[\frac{r_{\mathrm{f}}}{L_{0} \sigma_{0}^{m} \tau_{\mathrm{f}}(m+1)}\left\{\left(\sigma_{\mathrm{t}}-\frac{2 \tau_{\mathrm{f}} L_{\mathrm{d}}}{r_{\mathrm{f}}}\right)^{m+1}-\sigma_{\mathrm{t}}^{m+1}\right\}\right] .
$$

where $m$ and $\sigma_{0}$ are Weibull parameters. In this work, we substituted $m=4.7, \sigma_{0}=2400 \mathrm{MPa}$ and $L_{0}=25 \mathrm{~mm}$. They were determined by a tension test of the ECR-glass fiber.

Using these equations, we obtain bridging-stress with the debonding length and corresponding interfacial debonding energy, $\Gamma_{\mathrm{d}}$.

\subsection{Interfacial degradation}

$\Gamma_{\mathrm{d}}$ was obtained by fragmentation test ${ }^{(6)}$. The examinations were conducted for the ECR/vinyl ester specimen. In the fragmentation test, we obtained the relationship between the $\Gamma_{\mathrm{d}}$ and the immersion time $t_{\mathrm{i}}$ as shown in Fig. 7. And we obtained the moisture-concentration at fiber/matrix interface by the FEM calculation based on Fickian diffusion using the diffusion coefficient. Figure 7 shows also a relationship between the moistureconcentration distribution at the fiber/matrix interface and the immersion time $t_{\mathrm{i}}$. To apply the fragmentation result to the bridging mechanism in the DCDC test, we approximated the moisture-concentration as a function of the immersion time. The $\Gamma_{\mathrm{d}}$ distribution as a function of the moisture-concentration was obtained from Fig. 7.

Furthermore, in the DCDC specimen the moistureconcentration distribution was obtained as a function of 


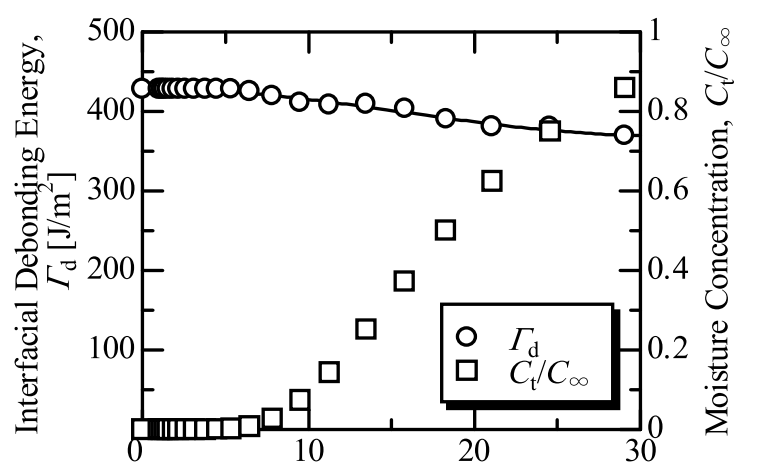

Root of Immersion Time, $t_{\mathrm{i}}^{0.5}$ [hour ${ }^{0.5}$ ]

Fig. 8 Interfacial debonding energy obtained by DCDC test as a function of root of immersion time and corresponding moisture concentration

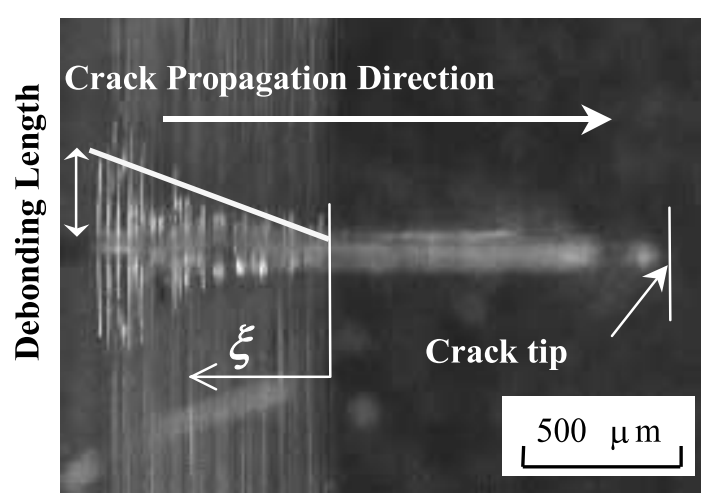

Fig. 9 Photograph of crack tip and interfacial debonding during DCDC test

the immersion time by conducting the FEM analysis as shown in Fig. 8. Using the two relationships, the relationship between the $\Gamma_{\mathrm{d}}$ and the immersion time in the DCDC test is described approximately as follows;

$$
\Gamma_{\mathrm{d}}=0.00339 t_{\mathrm{i}}^{3}+0.0194 t_{\mathrm{i}}^{2}-2.41 t_{\mathrm{i}}+432
$$

The $\Gamma_{\mathrm{d}}$ distribution as a function of the $t_{\mathrm{i}}$ is shown in Fig. 8. As shown in Fig. 8, FEM analysis showed that the moisture concentration increased after 6 hours $^{0.5}$. We can predict that the moisture caused the interfacial degradation after 6 hours ${ }^{0.5}$.

The interfacial debonding length $L_{\mathrm{d}}$ during the DCDC test was observed as shown in Fig. 9. The $L_{\mathrm{d}}$ was obtained as half the length of the white band. $L_{\mathrm{d}}$ distribution as a function of fiber position, $\xi$, is shown in Fig. 10. The origin of the $\xi$ and the direction are shown in Fig. 9, respectively. We performed the observations when the crack length was $6 \mathrm{~mm}$. As shown in Fig. 10, the interfacial debonding behavior in the ECR/vinyl ester was divided into two stages at 48 hours. After this time, the interfacial debonding energy decreased as shown in Fig. 8. Therefore, the approximation of the debonding energy distribution in the DCDC test can be considered appropriate. By substituting the values of $\Gamma_{\mathrm{d}}$ and $L_{\mathrm{d}}$ at each immersion time, we calculated $\sigma_{\mathrm{t}}$ using Eq. (5). Subsequently, on the

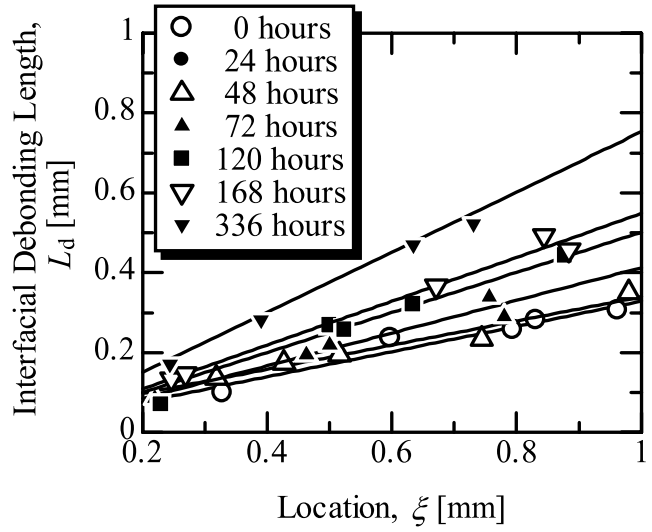

Fig. 10 Interfacial debonding length as a function of location

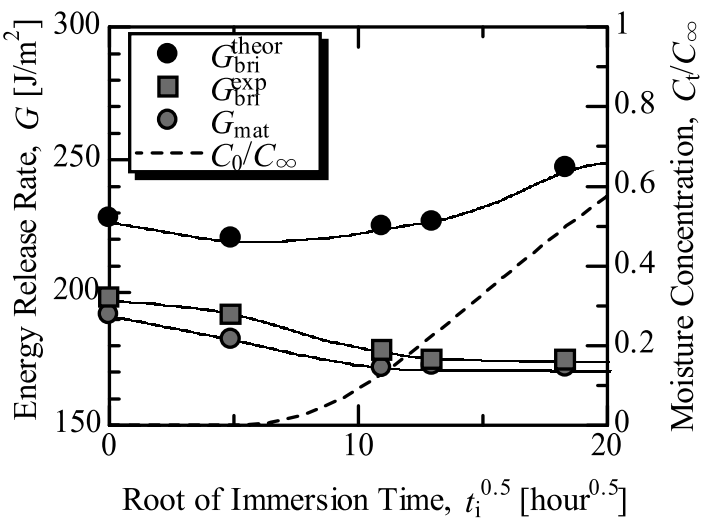

Fig. 11 Energy release rate of matrix and bridged specimen obtained experimentally and bridged specimen obtained theoretically based on interfacial debonding

basis of determination of $\sigma_{\mathrm{t}}$, we obtained the $\Delta G$ using Eq. (4). Finally, we obtained $G_{\text {bri }}=G_{\text {mat }}+\Delta G$ as shown in Fig. 11. The $G_{\text {bri }}$ obtained theoretically increased after 7 hours ${ }^{0.5}$. This result indicates that the increasing moisture caused a variation in the bridging behavior. One can predict that interfacial degradation will contribute to the enhancement of toughening by fiber bridging. The $\Delta G$ in the theoretical results differed from the experiments. In the DCDC specimen the interface was exposed in the water. Diffusivity along the interface was predicted to be extremely large compared with the matrix. Therefore, we predicted that interfacial degradation was promoted in the experiment. Consequently, the enhancement of toughening in the theoretical distribution differed from that in the experiment. The $\Delta G$ depends on the $\Gamma_{\mathrm{d}}$ as shown in Eqs. (4) and (5), and $\Gamma_{\mathrm{d}}$ converged as shown in Fig. 8. Therefore, the $G_{\text {bri }}$ is predicted to converge after 20 hours $^{0.5}$.

In the SCC, the crack tip is exposed in the environment. The $\Delta G$ under the water environment was determined using the interfacial debonding length and the moisture concentration. The results of our study indicate that the moisture-concentration distribution at the crack tip 
is required to predict the crack propagation behavior in the SCC.

\section{Conclusion}

The contribution of the interfacial degradation caused by the water on the fiber-bridging mechanism was measured for crack-propagation behavior under the water environment. As the result of the DCDC test, the decrease in the $G_{\text {mat }}$ depended on the weight gain due to the water uptake. Therefore, when the weight gain saturated, the decrease of $G_{\text {mat }}$ converged. Although the $G_{\text {mat }}$ of the epoxy specimen was larger than that of the vinyl ester specimen at an early stage of the immersion time in the water, the $G_{\text {mat }}$ of the vinyl ester specimen exceeded the epoxy specimen at the saturation level of the water uptake, because the saturation level of the epoxy was larger than the vinyl ester.

The interaction between bridging-fibers and the interfacial debonding on crack-propagation of the matrix was measured in the DCDC test. The variation in the bridging mechanism caused by water absorption was confirmed experimentally. The bridging contribution on the toughness in the ECR/vinyl ester specimen peaked at 5 hours $^{0.5}$. The toughness enhanced by the bridging was calculated based on microscopic mechanics with the measurements of interfacial debonding. Results of the calculation also showed that the toughening obtained by the bridging changed similarly to the results.

\section{References}

( 1 ) Price, J.N. and Hull, D., Effect of Matrix Toughness on Crack Propagation during Stress Corrosion of Glass Reinforced Composites, Composites Science and Technology, Vol.28, No.3 (1987), pp.193-210.

(2) Friedrich, K., Stress Corrosion Crack Propagation in Glass Fibre Reinforced /Thermoplastic PET, Journal of Material Science, Vol.16, No.12 (1981), pp.32923302.

( 3 ) Hogg, P.J., A Model for Stress Corrosion Crack Growth in Glass Reinforced Plastics, Composites Science and Technology, Vol.38, No.1 (1990), pp.23-42.
( 4 ) Hogg, P.J., Sapalidis, S.N. and Youd, S.J., High Temperature Acidic Stress Corrosion of Glass Fibre Composites, Journal of Material Science, Vol.32, No.2 (1997), pp.309-316.

( 5 ) Kawada, H., Okada, A., Ueno, H. and Hayashi, I., Stress Corrosion Cracking of Notched GFRP Laminates (Microscopic Fracture Model and Crack Propagation Rate), Trans. Jpn. Soc. Mech. Eng., (in Japanese), Vol.61, No.592, A (1995), pp.2566-2571.

( 6 ) Kawada, H. and Inoue, D., Study on Stress Corrosion Cracking o GFRP Using Single-Fiber Model Specimens (Evaluation of Interfacial Degradation by Energy-Balance Model), JSMS COMPOSITESVol.32 (2003), pp.75-78.

( 7 ) Bulter, E., Fuller, E.R. and Cai, H., Interactions of Matrix Cracks with Inclined Fibers, Ceram. Eng. Sci. Proc., Vol.13 No.7-8 (1992), pp.475-482.

( 8 ) Coyle, T.W., Fuller, E.R. and Swanson, P., Fracture Mechanics Characterization of Crack/Fiber Interactions in Ceramic Matrix Composites, Ceram. Eng. Sci. Proc., Vol.8, No.7-8 (1987), pp.630-635.

( 9 ) Sun, Y. and Singh, R.N., Determination of Fiber Bridging Stress Profile by Debond Length Measurement, Acta Materialia, Vol.48, No.13 (2000), pp.3607-3619.

(10) $\mathrm{Xu}$, G., Bower, A.F. and O.ritz, M., The Influence of Crack Trapping on the Toughness of Fiber Reinforced Composites, J. Mech. Phys. Solids, Vol.46, No.10 (1998), pp.1815-1833.

(11) Janssen, C., Specimen for Fracture Mechanics Studies on Glass, Proceedings of the Xth International Congress on Glass, Kyoto, Japan, July 8-13, (1974), pp.23-30.

(12) He, M.Y., Turner, M.R. and Evans, A.G., Analysis of the Double Cleavage Drilled Compression Specimen for Interface Fracture Energy Measurements over a Range of Mode Mixities, Acta Metallurgica et Materialia, Vol.43, No.9 (1995), pp.3453-3458.

(13) Ritter, J.E., Fox, J.R., Hutko, DI. and Lardner, T.J., Moisture-Assisted Crack Growth at Epoxy-Glass Interfaces, Vol.33, No.19 (1998), pp.4581-4588.

(14) Oh, H.L. and Finnie, I., On the Location of Fracture in Brittle Solids-I Due to Satic Loading, International Journal of Fracture Mechanics, Vol.6 (1970), pp.287300 . 